\title{
nature
}

\section{Why sequence the human genome?}

General enthusiasm for producing a sequence of the nucleotides in the whole of human DNA is well justified, but more attention should be paid to the need for an international data-gathering network.

THE support for ambitions to sequence the human genome provided last week by the US National Research Council (the investigative arm of the national academies of science, medicine and engineering) is predictably intelligent, but may nevertheless help to make an institution of a project that should be much more informally part of the scientific community's endeavour. So much, at least, should be clear from the way in which the committee under Dr Bruce Alberts (see p. 467) considered which of several US government agencies should be the "lead agency" in Washington-speak - and then failed to agree on a solution. The obvious danger is that some inter-agency committee will now take over from where the Alberts committee left off, will bang a few heads together, nominate one or other of the likely candidates and then set about the building of an organization in the mould of the Apollo project.

This is not to say that the sequencing of the human genome is not a worthwhile goal. On the contrary, it is every bit as important as the Alberts committee says. The great savings of effort (and thus of money) that would derive from ready access to a sequence of the nucleotide bases in the human genome are easily understood, perhaps most readily in the continuing search for the genetic basis of inherited disease, but are far from being the chief benefits of a successful sequencing project. It is at least as important that a secure understanding of the function of the apparently redundant DNA in the genomes of higher organisms - is it meaningful, or the consequence of evolutionary history and thus 'junk'? - will not be possible without the complete sequencing of some representive genomes. Another prize, still a long way off, is what will be learned from the inspection of a complete genome about the way in which that genome functions, with sequential and/or selective activation of specific genes. But the Alberts committee is right to draw attention to the importance of comparing the genomes of different species; not only will this advance the identification of functional genes in the sequenced genomes, but the understanding of evolutionary relationships that will also emerge may ultimately be the greatest benefit of efforts now being mounted.

\section{Milestone}

Unfortunately, the rhetoric of the past several months has falsely created the impression that the project to sequence the human genome is a self-contained and one-off enterprise - a task with a fixed end-point and thus a restricted (if very large) cost (which is why it appeals to those who manage public budgets and their reception by bodies such as the US Congress). The truth is that even such a specific and substantial task should be regarded as but another milestone, still in the future, in the accumulation of knowledge about the real world. Moreover, it would be folly to suppose that the sequencing business will be worked out, and that molecular biologists can turn their attention to other things, once the sequence of nucleotides in human DNA is neatly tidied away in some computer memory. Those considering what organizations should do the job had better look beyond the immediate goal. That is especially so as there is no shortage of talented people willing to do the job.

What should the public grant-making agencies be doing in the circumstances? The first need is for support with the development of automatic sequencing techniques, to which government agencies in the United States and Japan have already responded. These initiatives, a necessary preliminary to the human genome project, are nevertheless important in their own right. It will very soon be as pointless that a graduate student's success should be judged by the length of the sequence he presents in the appendix to his thesis as proof of having built a number of amplifiers has long-ceased to be a test of competence in nuclcar physics. Moreover, the full benefits that will flow in diagnostic medicine from automatic and rapid techniques of sequencing short pieces of the human genome still cannot be guessed at. The $\$ 17$ million being spent this year in the United States on these developments will be a sound investment.

Less has been heard of the other urgent need, that for the improvement of the existing databanks of nucleotide sequences, and for the development of novel data-handling techniques. The existing databanks, in the United States and Europe, are already overwhelmed by the present relatively meagre flow of data, much of which is stored without adequate annotation. How would they be able to handle the information that will be available when the even the first instalment of the US investment in automation bears fruit? As things are, there are no arrangements for making sure that the fullest information is wrung from such nucleotide sequences as they find their way to the databanks, but it would plainly be a considerable economy of efforts already expended if the databanks were equipped to answer questions that currently require experimental investigation - to which human chromosome does a notified sequence belong, for example? A further anomaly is that the formal databanks are equipped to handle nucleotide sequences, but that responsibility for maintaining the coarser-scale physical maps of various genomes rests with the academic groups who have made them in the first place. Over this confusing scene is the question of whether, when the sequence of some version of the human genome is successfully stored in a machine, people with a legitimate interest will be able to ask intelligent questions of it.

The moral to be drawn from this state of affairs is that those seeking to create organizations should turn the problem of handling data into an opportunity. If the existing databanks were a necessity when they were first created five years ago, improved versions of them are surely even more necessary now that they are in danger of becoming the victims of their own success. This is where the organization-building should begin. Moreover, as must be plain, the job should be tackled internationally so as to win the greatest benefits from the data gathered in widely scattered laboratories. The ideal would be that such a project should be mounted in a way that commands the respect of the research community. Although the costs would ultimately have to be met by national grant-making agencies, the notion that there might be charges for information supplied should not be excluded. The obvious snag is that the creation of such an organization is bound to take time. But that is merely an argument for starting soon on building an organization without which the human genome project might be a fruitless excrcise. 